\title{
Numerical Simulation of Free Convection and Surface Radiation in a Large-Scale Cavity with a Heater
}

\author{
Igor Miroshnichenko, Mikhail Sheremet, Stepan Mikhailenko \\ Tomsk State University \\ 36 Lenina Avenue, Tomsk, Russia \\ miroshnichenko@mail.tsu.ru; sheremet@math.tsu.ru, stepanmihaylenko@gmail.com
}

\section{Extended Abstract}

Numerical and experimental studies of turbulent natural convection in closed rectangular enclosures represent a highly actual problem for investigators due to its great applicability in various technological applications [1-3]. One important application of this type of geometry is heat transfer in rooms and buildings. For construction and design of houses one of the important goals is to provide thermal comfort for occupants. Moreover, it's also a requirement for buildings to be energy efficient. Recent developments in processing power and algorithms make it possible to simulate real physical processes at levels of detail that were almost impossible just a few years ago. In this connection, the numerical simulation of convective-radiative heat transfer has received considerable attention from many researchers.

The aim of the present study is to investigate numerically the turbulent modes of the conjugate natural convection combined with surface radiation in a large enclosure bounded by solid walls with a heat-generating element. A heater and solid walls were assumed to be gray, opaque and diffuse emitters. The Boussinesq approximation was used to describe the density changes. Mathematical model has been formulated in terms of the dimensionless stream function, vorticity and temperature. The finite-difference method is used to solve the set of governing equations. To change a non-uniform grid in physical domain to a uniform grid in computational domain a special algebraic coordinate transformation has been applied. The developed computational code has been validated successfully using the following numerical results [4-5].

In order to study the effect of radiation on thermal transmission, the total heat transfer from the heater was evaluated using the average radiative and convective Nusselt numbers. It was found that the contribution of radiation is a significant topic for energy transport in closed enclosures. An increase in surface emissivity of internal surfaces leads to a growth of the average radiative Nusselt number, while the convective average Nusselt number is insignificantly changed. Furthermore, a growth of surface emissivity of internal surfaces allows us to reduce the average temperature inside the heater and, at the same time, leads to a slight increase in the mean temperature inside the enclosure.

This work was supported by the Russian Science Foundation (Project No. 19-79-00296).

\section{References}

[1] F. Ampofo, "Turbulent natural convection of air in a non-partitioned or partitioned cavity with differentially heated vertical and conducting horizontal walls," Exp. Therm. Fluid Sci., vol. 29, pp. 137-157, 2005.

[2] T. Fusegi, "Turbulent natural convection in a sidewall-heated cavity using a two-equation model of temperature," Int. J. Numer. Method H., vol. 4, pp. 483-496, 1994.

[3] A.K. Sharma, K. Velusamy, C. Balaji, "Turbulent natural convection in an enclosure with localized heating from below," Int. J. Thermal Sci., vol. 46, pp. 1232-1241, 2007.

[4] H. Wang, S. Xin, P. Le Quere, "Numerical study of natural convection-surface radiation coupling in air-filled square cavities", C.R. Mecanique, vol. 334, pp. 48-57, 2006.

[5] C. Zhuo, C.Zhong, "LES-Based Filter-Matrix Lattice Boltzmann Model for Simulating Turbulent Natural Convection in a Square Cavity", Int. J. Heat Fluid Flow, vol. 42, pp.10-22, 2013. 